\title{
Controlled Double-Jet Mixing of Light Speeds in the Trigonometric Realm
}

\author{
Jiří Stávek ${ }^{1}$ \\ ${ }^{1}$ Bazovského 1228, 16300 Prague, Czech republic \\ Correspondence: Jiří Stávek, Bazovského 1228, 16300 Prague, Czech republic. E-mail: stavek.jiri@seznam.cz
}

Received: January 10, 2018

Accepted: January 27, 2018

Online Published: January 30, 2018

doi:10.5539/apr.v10n1p53

URL: https://doi.org/10.5539/apr.v10n1p53

\begin{abstract}
We were inspired by three great concepts: 1 . Pythagorean means, 2. Pierre de Fermat's principle of the least time, 3. Michael Faraday's concept of vibrating rays in longitudinal and transverse directions. We have formulated an extended Doppler formula for rectilinear motions in the absolute spacetime where both postulates of the special relativity are valid. The second postulate of the special relativity for rectilinear motions was derived as the harmonic mean speed based on the Pythagoras-Fermat-Faraday $\left(\mathrm{PF}^{2}\right)$ model. The null result of the MichelsonMorley experiment is valid for the arms of that interferometer separated by the angle $\pi / 2$. However, for the angles smaller than $\pi / 2$ a predicted fringe shift should be observed. For the circular motions the Doppler formula combines the longitudinal and transverse speeds in such a way that the frequency of the rotating light beam is diluted by a factor $\left[1-(\mathrm{v} / \mathrm{c})^{2}\right]^{0.5}$ and the wavelength of that light beam is extended by the same factor. The Doppler formula for light beams can be tested for the rotating source and the detector placed close to the rim of that rotating disk in a defined position. In order to obtain new experimental data we propose to construct the Michelson-Morley-HarressSagnac interferometer where rotating disks have being attached to both arms of the Michelson interferometer. In rotating disks we might prepare light beams with defined independent values of their longitudinal speeds and after the mixing of these two light beams on their return path to the detector we might observe predicted fringe shifts. In these circular paths the second postulate of the special relativity is not valid. The full composition of Doppler formula is given by the interplay of the macro Doppler effect (the relative motion of the source and observer) and the micro Doppler effect (the combination of the longitudinal and transverse vibration speeds of that oscillating particle - the elasticity of the photon wave).
\end{abstract}

Keywords: Pythagorean means, Fermat's principle of the least time, Faraday's concept of vibrating rays, extended Doppler formula for rectilinear and circular motions, controlled double-jet mixing of light speeds, micro Doppler effect, macro Doppler effect

\section{Introduction}

The famous quote of Heraclitus "Nature loves to hide" was described in details by Hadot (2008). Hadot in his valuable book gives us many examples how Nature protects Her Secrets. In some cases it desires an enormous research of many generations before the right "recipe" unlocking the true reality can be found. Therefore, we should steadily experimentally test our models in order to avoid "blind alloys". In the last century the veil called as the elastic spacetime was intensively experimentally studied and almost all researchers concluded that it is the final true veil of Nature.

In our contribution we attempt to find a more general Doppler formula with the aim to penetrate through the veil of the elastic spacetime. In our approach we have combined three valuable sources of Old Masters: 1 . Pythagorean means, 2. Pierre de Fermat's principle of the least time, 3. Michael Faraday's concept of vibrating rays.

Ancient Greeks used Pythagorean means to describe average values of two numbers: the harmonic mean, the geometric mean and the arithmetic mean. These Pythagorean means should be used according the context on that studied situation.

Pierre de Fermat introduced his principle of the least time that described many natural processes with light beams. We will use this principle for light beams that should travel a longer path with the highest possible speed while the shorter paths with the lowest possible speed. Probably, the first attempt to use this Fermat's principle for the description of the Sagnac effect was made by Harzer (1920). 
Faraday's lecture "Thoughts on Ray Vibrations" presented in London on $3^{\text {rd }}$ April 1846 influenced many researchers and stimulated the formation of valuable models. This concept several time appeared and disappeared from the focus of researchers (e.g., for references see Bilbao et al., 2014). All these activities were stopped by the second postulate of the special relativity - vibrating rays models based on Galilean speed additions were experimentally falsified.

In our "recipe" we have mixed ingredients of Pythagoras-Fermat-Faraday $\left(\mathrm{PF}^{2}\right)$ in order to describe a more general Doppler formula that might be experimentally tested both in rectilinear and circular motions. Moreover, we have described the second postulate of the special relativity as the harmonic mean speed of longitudinal vibrations of the oscilating particle for the rectilinear motions. The full composition of Doppler formula is given by the interplay of the macro Doppler effect (the relative motion of the source and observer) and the micro Doppler effect (the combination of the longitudinal and transverse vibration speeds of that oscillating particle - the elasticity of the photon wave).

\section{Structure of the Doppler Formula}

The Doppler effect is the change in frequency and wavelength of a wave for an observer who is moving relative to the wave source. Christian Doppler described this phenomenom in Prague on 25 $5^{\text {th }}$ May 1842:

$$
\frac{\nu_{\text {source }}}{\nu_{\text {observer }}}=\frac{\lambda_{\text {observer }}}{\lambda_{\text {source }}}=1+\frac{v}{c} \cos \theta
$$

For the source receding from the observer $\cos \theta=1$, for the source approaching $\cos \theta=-1$. We will describe this part of the Doppler formula as the macro Doppler effect - the relative motion between the source and its observer.

Albert Einstein in 1905 based on the concept of the elastic spacetime added one very important factor "time dilation" to this Doppler formula:

$$
\frac{\nu_{\text {source }}}{\nu_{\text {observer }}}=\frac{\lambda_{\text {observer }}}{\lambda_{\text {source }}}=\frac{1}{\sqrt{1-\frac{v^{2}}{c^{2}}}}\left(1+\frac{v}{c} \cos \theta\right)
$$

This formula describes perfectly the frequency and wavelength of light waves emitting from moving source in many situations. However, there are still several redshift anomalies. Moreover, we have to give up the 3D space and accept the concept of the elastic spacetime. In our attempt we will assume that this factor describes partly the elasticity of the photon wave - the micro Doppler effect.

Can we find a modification of the Doppler formula in order to explain elasticity of the light waves in the 3D space?

Michael Faraday proposed in his lecture "Thoughts on Ray Vibrations" that light could not be the result of aether vibrations but vibrations of the physical lines of force in the longitudinal and transverse directions. So, we might attempt to extend the Doppler formula by the ratio of the longitudinal vibration speed and the transverse vibration speed of an oscilating particle:

$$
\frac{V_{\text {source }}}{V_{\text {observer }}}=\frac{\lambda_{\text {obsener }}}{\lambda_{\text {source }}}=\frac{\text { longitudinal vibration speed }}{\text { transverse vibration speed }}\left(1+\frac{v}{c} \cos \theta\right)
$$

These simultaneous longitudinal and transverse vibrations of particles could be modeled as the combination of harmonic oscillation of the Hooke's spring and the Galilean pendulum. Another analogy for this situation could be the motion of locomotive wheels. Locomotive wheels spin at a frequency $v$ cycles per second and the mechanical linkages allow the linear vibration of the steam engine's pistons at the frequency $v$ in the rectilinear direction. The ratio of the longitudinal and trasverse vibration speeds describes the elasticity of the photon wave the micro Doppler effect. 


\section{Supporting Trigonometric Relations}

For the proposed $\mathrm{PF}^{2}$ model several trigonometric relations were used. These relations are given in the Table 1 and in Figures 1 and 2.

Table 1. Trigonometric relations for the defined $\cos \varepsilon=\mathrm{v} / \mathrm{c}$

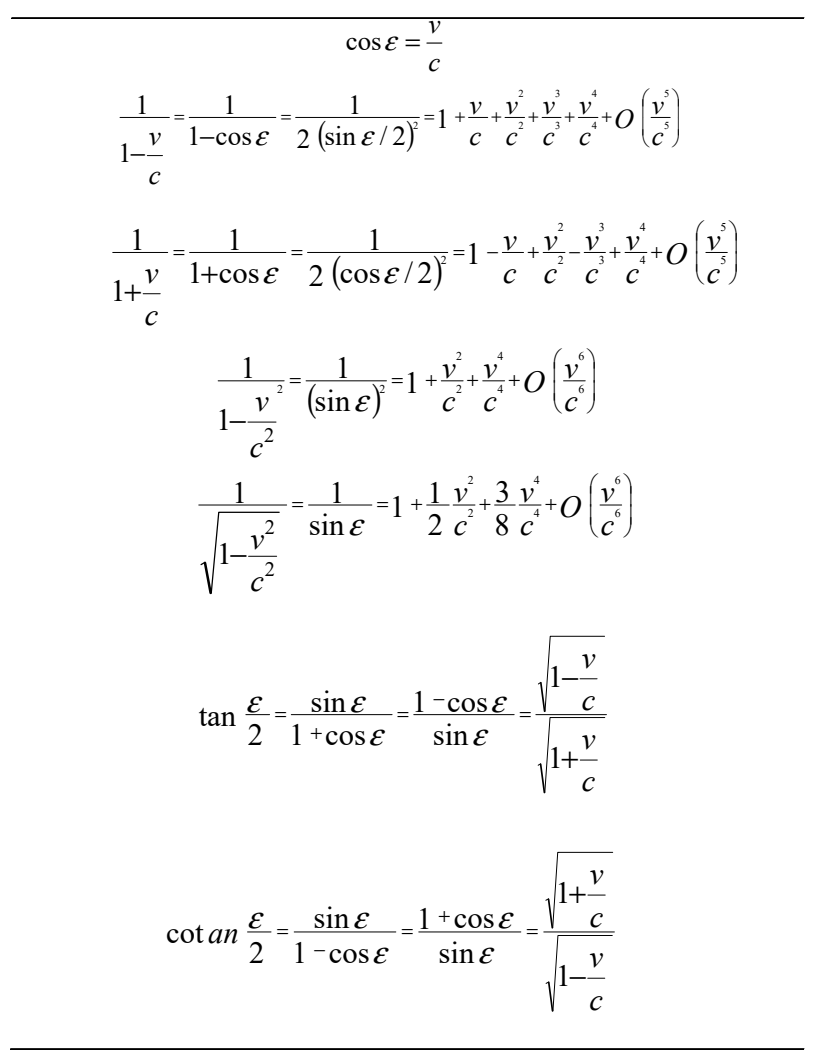

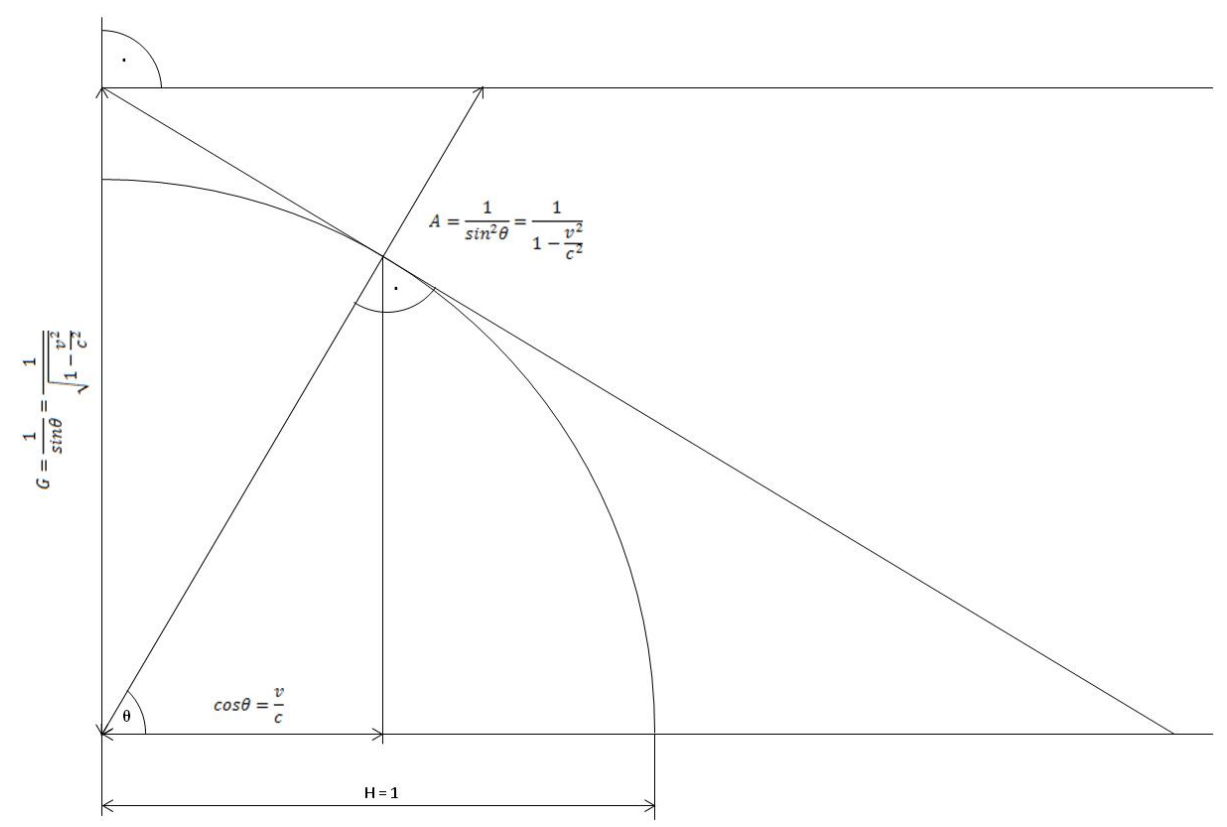

Figure 1. Pythagoras-Fermat-Faraday mean speeds: H - harmonic mean speed, G - geometric mean speed, A arithmetic mean speed if we define $\cos \theta=\mathrm{v} / \mathrm{c}$ 


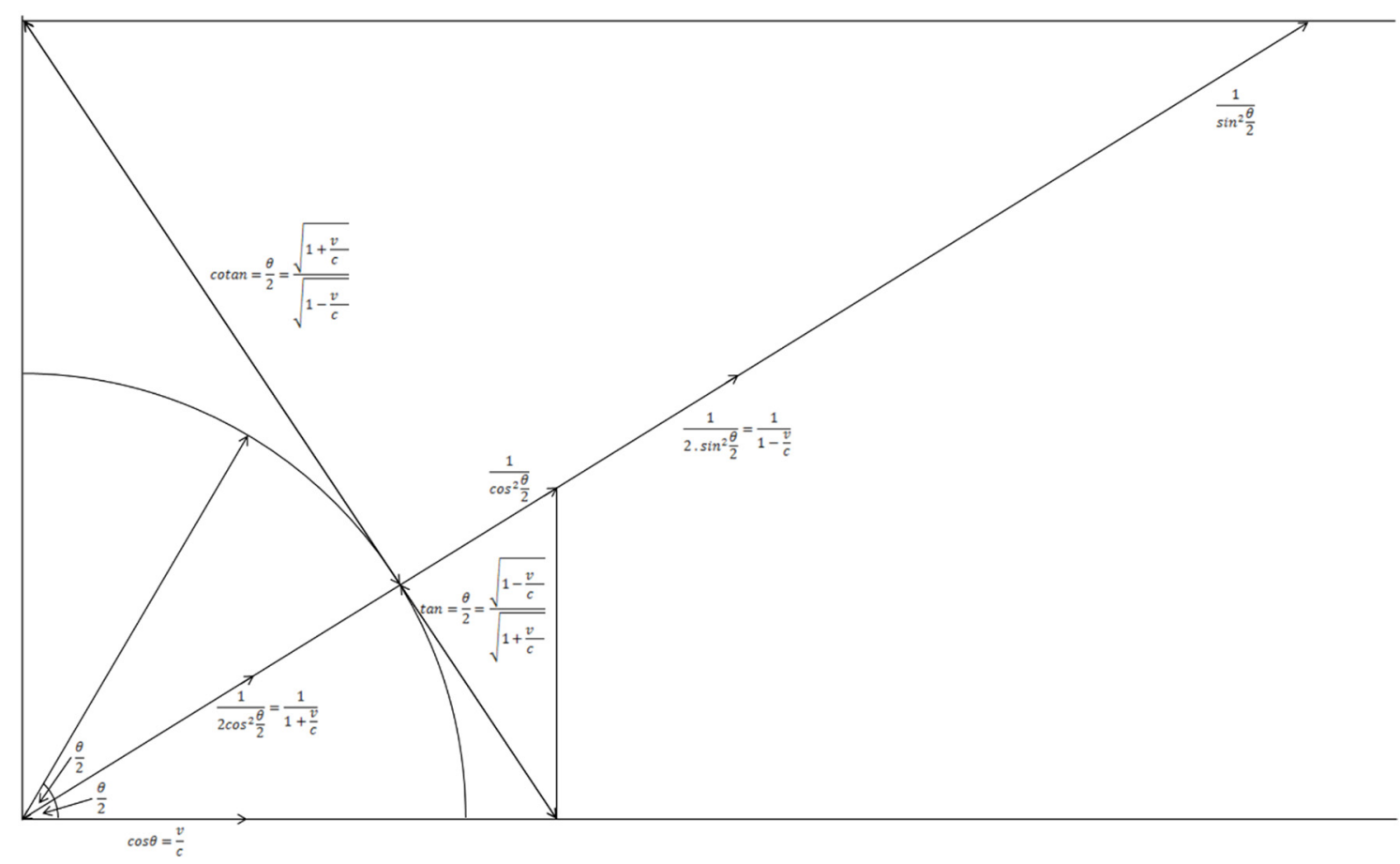

Figure 2. Some trigonometric functions for longitudinal and transverse speeds if we define $\cos \theta=\mathrm{v} / \mathrm{c}$

\section{Pythagoras-Fermat-Faraday $\left(\mathrm{PF}^{2}\right)$ Mean Speeds for Rectilinear Motions}

We will combine two speeds $\mathrm{c}_{1}=\mathrm{c} /(1+\mathrm{v} / \mathrm{c})$ and $\mathrm{c}_{2}=\mathrm{c} /(1-\mathrm{v} / \mathrm{c})$ according the rules for harmonic, geometric and arithmetic mean speeds. The speed $c_{1}$ should be used for shorter paths, the speed $c_{2}$ should be used for longer paths in order to fulfill the Fermat's principle of the least time.

$\mathrm{PF}^{2}$ harmonic mean speed describes longitudinal vibration speed of photons leaving their source - the second postulate of the special relativity valid for rectilinear motions:

$$
c_{H}=\frac{2}{\frac{1}{c_{1}}+\frac{1}{c_{2}}}=c
$$

$\mathrm{PF}^{2}$ geometric mean speed describes the transverse vibration speed of photons for the angle $\theta=0$ :

$$
c_{G}=\sqrt{c_{1} c_{2}}=\frac{c}{11-\frac{v^{2}}{c^{2}}}
$$

$\mathrm{PF}^{2}$ arithmetic mean speed describes the longitudinal vibration speed of photons at the observer for the angle $\theta=$ 0 :

$$
c_{A}=\frac{c_{1}+c_{2}}{2}=\frac{c}{\left(1-\frac{v^{2}}{c^{2}}\right)}
$$

Equation 7 describes the elasticity of the photon wave during the rectilinear motions: the first part represent the transverse vibration speed, the second part the longitudinal vibration speed and the third part is the macro Doppler effect: 


$$
\frac{\nu_{\text {source }}}{V_{\text {observer }}}=\frac{\lambda_{\text {obsener }}}{\lambda_{\text {source }}}=\frac{\sqrt{1-\frac{v^{2}}{c^{2}} \cos ^{2} \theta}}{1} \frac{\sqrt{1-\frac{v^{2}}{c^{2}} \sin ^{2} \theta}}{1-\frac{v^{2}}{c^{2}}}\left(1+\frac{v}{c} \cos \theta\right)
$$

This extended Doppler formula for rectilinear motions describes the elasticity of photon waves on the dependence of the angle $\theta$ between the motion of the source and the observer. For rectilinear motions with the relative angles $0<\theta<\pi / 2$ we should find small differences in the Doppler effect in compare with the prediction of the special relativity.

We can test the predictions of this formula for Michelson-Morley experiment for the interferometer arms separated by the angle $\theta=\pi / 2$. In this case there is no relative motion between the source and the detector - there is no contribution of the macro Doppler effect. We can observe only the contribution coming from the elasticity of the photon wave - the micro Doppler effect.

The observed fringe shift for this condition should be (arms of the interferometer are separated by the angle $\theta=$ $\pi / 2)$ :

$$
\Delta n=\frac{2 L}{\lambda}\left[\frac{\sqrt{1-\frac{v^{2}}{c^{2}} \cos ^{2} \frac{\pi}{2}} \frac{\sqrt{1-\frac{v^{2}}{c^{2}} \sin ^{2} \frac{\pi}{2}}}{1}}{1-\frac{v^{2}}{c^{2}}}-\frac{\sqrt{1-\frac{v^{2}}{c^{2}} \cos ^{2} 0}}{1} \frac{\sqrt{1-\frac{v^{2}}{c^{2}} \sin ^{2} 0}}{1-\frac{v^{2}}{c^{2}}}\right]=0
$$

We can test the predictions of this formula for Michelson-Morley experiment for the interferometer arms separated by the angle $\theta=\pi / 4$. The observed fringe shift for this condition should be:

$$
\begin{aligned}
& \Delta n=\frac{2 L}{\lambda}\left[\frac{\sqrt{1-\frac{v^{2}}{c^{2}} \cos ^{2} \frac{\pi}{4}}}{1} \frac{\sqrt{1-\frac{v^{2}}{c^{2}} \sin ^{2} \frac{\pi}{4}}}{1-\frac{v^{2}}{c^{2}}}-\frac{\sqrt{1-\frac{v^{2}}{c^{2}} \cos ^{2} 0}}{1} \frac{\sqrt{1-\frac{v^{2}}{c^{2}} \sin ^{2} 0}}{1-\frac{v^{2}}{c^{2}}}\right]= \\
& =\frac{2 L}{\lambda}\left[\frac{1-\frac{1}{2} \frac{v^{2}}{c^{2}}}{1-\frac{v^{2}}{c^{2}}}-\frac{1}{\sqrt{1-\frac{v^{2}}{c^{2}}}}\right] \cong \frac{2 L}{\lambda} \frac{0.125 v^{4}}{c^{4}}
\end{aligned}
$$

We have obtained the fourth order formula and such small fringe shifts could be measured by the only one existing interferometer - the LIGO interferometer. We assume that the first postulate of the special relativity is valid also for the absolute spacetime then we expect the fringe shift on the level of $\mathrm{v}^{4} / \mathrm{c}^{4} \approx 10^{-16}$. We can increase the fringe shift by the active length $L$ of the interferometer arms and by the longer wavelength $\lambda$ of the used laser source. To get more experimental data we can vary the angle between those two arms from 0 to $\pi / 2$.

\section{Pythagoras-Fermat-Faraday $\left(\mathbf{P F}^{2}\right)$ Mean Speeds for Circular Motions}

The Harress-Sagnac effect can be described both classically and relativistically. On both sides of this discussion many valuable papers were published - see the Reference list.

The behaviour of light beams on the circular path differs from the rectilinear path. We assume that on the circular path the photon does not vibrate in the longitudinal direction and we can observe the longitudinal speed according the Fermat's principle of the least time: for the shorter path the slowest possible longitudinal speed and for the longer path the highest possible longitudinal speed. The valuable inspiration we have found in the contributions of Walther Ritz (1908, 1909), Hans Witte (1914), Paul Harzer (1920), Stefan Marinov (1974) Franco Selleri (1996, 2004), S.J.G. Gift (2013-2015), and Sergey N. Artekha et al. (2017).

The longitudinal speed against the direction of rotation of the disk (the shorter path): 


$$
c_{1}=\frac{c}{1+\frac{v}{c}}
$$

The longitudinal speed in the direction of rotation of the disk (the longer path):

$$
c_{2}=\frac{c}{1-\frac{v}{c}}
$$

The transverse speed against the direction of rotation of the disk:

$$
c_{3}=\frac{1-\frac{v}{c}}{\sqrt{1-\frac{v^{2}}{c^{2}}}} c
$$

The transverse speed in the direction of rotation of the disk:

$$
c_{3}=\frac{1+\frac{v}{c}}{\sqrt{1-\frac{v^{2}}{c^{2}}}} c
$$

In the case of the rotation of that disk photon waves elastically react according the direction of rotation of the disk. Micro Doppler effect - oscillating particle travelling in the direction of the rotation of disk could be described as:

$$
\frac{\nu_{\text {source }}}{\nu_{\text {observer }}}=\frac{\lambda_{\text {observer }}}{\lambda_{\text {source }}}=\frac{\sqrt{1-\frac{v^{2}}{c^{2}}}}{1+\frac{v}{c}} \frac{1}{1-\frac{v}{c}}=\frac{1}{\sqrt{1-\frac{v^{2}}{c^{2}}}}
$$

Micro Doppler effect - oscillating particle travelling against the direction of the rotation of disk could be described as:

$$
\frac{\nu_{\text {source }}}{\boldsymbol{\nu}_{\text {observer }}}=\frac{\lambda_{\text {observer }}}{\lambda_{\text {source }}}=\frac{\sqrt{1-\frac{v^{2}}{c^{2}}}}{1-\frac{v}{c}} \frac{1}{1+\frac{v}{c}}=\frac{1}{\sqrt{1-\frac{v^{2}}{c^{2}}}}
$$

The composition of the longitudinal speed and transverse speed of photons gives for both directions of the disk rotation the very well known "time dilation" introduced by Albert Einstein in 1905 based on the elasticity of the spacetime. However, in our model the "time dilation" is caused by the combination of the transverse vibration of an oscillating particle and its longitudinal speed. If both the source and the detector rotate on the disk we can observe the micro Doppler effect only, no macro Doppler effect could be observed.

If we place the source of the light beam on the rotating disk and the detector outside of that rotating disk in a defined position we should observe both the micro Doppler effect and the macro Doppler effect. The valuable experiments for a sound source moving in a circle were done by Saba and Rosa (2003) who observed "The Doppler effect of a sound source moving in a circle". Son, Kwon and Choi (2014) found a more general solution of the Doppler effect of a sound source moving in a circle with a detector outside of that rotating disk - see Figure 3 . 


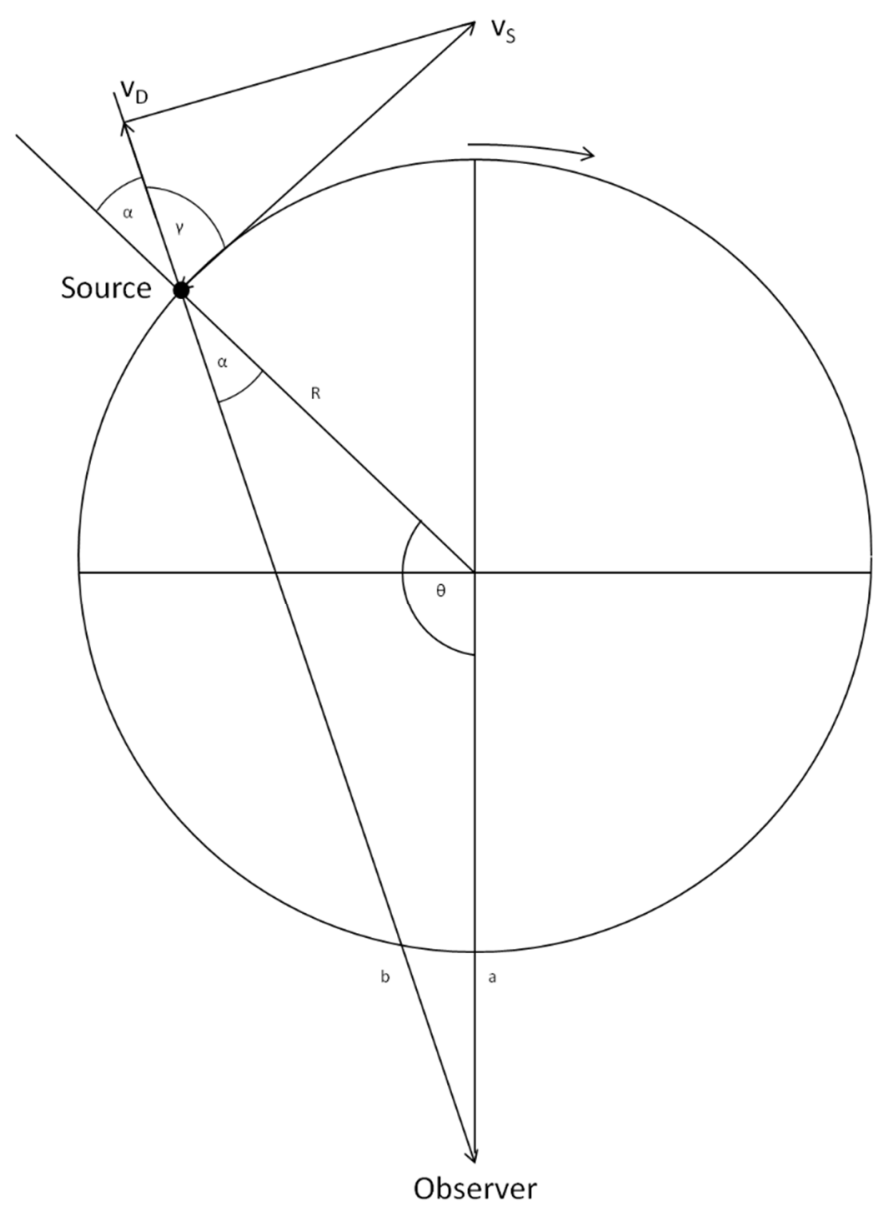

Figure 3. Light source moving in circle and an observer at a defined position outside of the rotating disk (based on the paper of Son, Kwon and Choi (2014)

In this Figure 3 the light source is moving in a circle with its tangential speed v/c, the observer is at a distance a from the centre of that roating disk and in a distance $\mathrm{b}$ from the rotating source. $\mathrm{R}$ is the radius of that disk and $\theta$ is the angle describing the relative position of the source to the observer.

$$
\frac{V_{\text {source }}}{V_{\text {obsener }}}=\frac{\lambda_{\text {obsener }}}{\lambda_{\text {source }}}=\frac{1}{\sqrt{1-\frac{v^{2}}{c^{2}}}}\left(1+\frac{v a \sin \theta}{\sqrt{a^{2}+R^{2}-2 a R \cos \theta}}\right)
$$

We have now the identical Doppler formula with the Einstein formula. How to distinguish experimentally the concept of the elastic spacetime and the concept of the elastic photon wave?

\section{Controlled Double-Jet Mixing of Light Speeds}

Since the publication of experimental data of Franz Harres (1912) and George Sagnac (1913) there is a very active discussion between the proponents of the classical physics and the proponents of the relativistic school.

In order to bring some new experimental data that might falsify one of those concepts we propose to construct the Michelson-Morley-Harress-Sagnac (M-M-H-S) interferometer. In this case we propose to attach rotating disks to both arms of the Michelson interferometer. We might manipulate independently with the speed of light beams in those rotating disks. On the return path to the detector both light beams are mixed and we might obtain a predicted fringe shift. The schema of this M-M-H-S interferometer is given by Figure 4. 


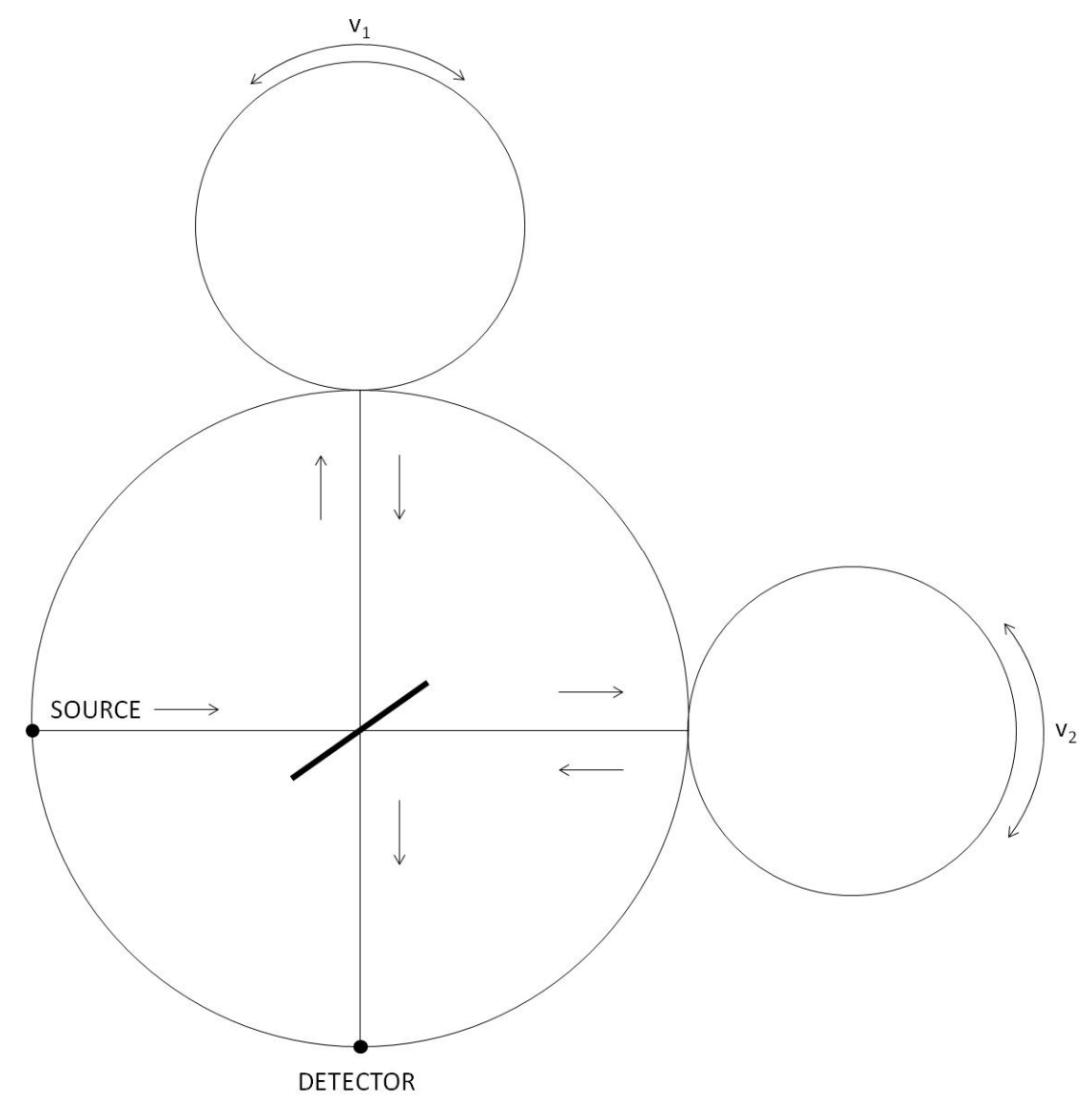

Figure 4. Michelson-Morley-Harress-Sagnac interferometer for the controlled double-jet mixing of light speeds

The predicted fringe shift for light beams travelling through rotating disks with radius $\mathrm{R}$, tangential speed of the roating disk 1 with $\mathrm{v}_{1}$, tangential speed of the rotating disk 2 with $\mathrm{v}_{2}$ :

$$
\Delta t=2 \pi R\left(\frac{1+\frac{v_{1}}{c}}{c} \mp \frac{1-\frac{v_{2}}{c}}{c}\right)=2 \pi R\left(\frac{\frac{v_{1}}{c} \pm \frac{v_{2}}{c}}{c}\right)
$$

For the period of the monochromatic light beam used $\tau=\lambda / \mathrm{c}$, the fractional displacement of the fringes, given by $\Delta \mathrm{N}=\Delta \mathrm{t} / \tau$ is:

$$
\Delta N=\frac{2 \pi R}{c \lambda}\left(v_{1}{ }^{ \pm} v_{2}\right)
$$

In this experimental arrangement it is possible to change independently both the value of the light speed and the direction of rotation of those rotating disks. The resulting mixture of light beams should create the predicted fringe shift. In this experimental arrangement we should observe the macro Doppler effect.

The final answer should be given by Nature as Peter Marquardt in 2013 deciphered the acronym S.A.G.N.A.C.: Science Advances Giving Nature All Credit.

\section{Conclusions}

1) The extended Doppler formula was derived as two contributions: the macro Doppler effect (the relative motion of the source and the observer) and the micro Doppler effect (the elasticity of the photon wave).

2) The second postulate of the special relativity was derived as the harmonic mean speed of the longitudinally oscillating particle.

3) For rectilinear motions with the relative angles $0<\theta<\pi / 2$ we should find small differences in the Doppler 
effect in compare with the predictions of the special relativity.

4) For rectilinear motions the micro Doppler effect was predicted for the Michelson interferometer with two arms separated by the angle less than $\pi / 2$.

5) For the circular motions of both the light source and the detector on the rotating disk the micro Doppler effect was derived - it is identical with the very-well known Voigt's transformation.

6) For circular motions of the light source the macro Doppler effect was predicted for the observer located outside of the rotating disk in a defined position.

7) For circular motions the macro Doppler effect was predicted for the case of the Michelson-Morley-HarressSagnac interferometer - Controlled Double-Jet Mixing of Light Speeds.

\section{Acknowledgments}

This work was supported by the JP\&FŠ Agency (Contract Number 25g/1963), by the VZ\&MŠ Agency (Contract Number 16000/1989) and by the GMS Agency (Contract Number 69110/1992). We have found the valuable support on the web site www.wolframalpha.com with the corrections of used formulae.

\section{References}

Anderson, R., Bilger, H. R., \& Stedman, G. E. (1994). Sagnac Effect: A Century of Earth-rotating Interferometers. Am. J. Phys., 62, 975-985.

Artekha, S. N., Chubykalo, A., \& Espinoza, A. (2017). On the Question of the Invariance of the Light Speed. Journal of Modern Physics, 8(08), 1213-1233. https://doi.org/10.4236/jmp.2017.88080

Bilbao, L., Bernal, L., \& Minotti, F. (2014). Vibrating Rays Theory. Retrieved from arxiv.org/abs/1407.5001

Bouyer, P. (2014). The Centenary of Sagnac Effect and Its Applications: from Electromagnetic to Matter Waves. Gyroscopy and Navigation, 5, 20-26.

Doppler, C. (1842). Über das farbige Licht der Doppelsterne und einiger anderer Gestirne des Himmels. Abhandlungen der Königl. Böhm. Gesselschaft der Wissenschaften, V. Folge, Bd., 2, 465-482.

Dufour, A., \& Prunier, F. (1942). Sur un déplacement de franges enregistré sur une plate-forme en rotation uniforme. Le J. de Ph. et le Rad., 3, 153-161.

Einstein, A. (1905). Zur Elektrodynamik bewegten Körpern. Ann. Physik, 17, 891-921.

Einstein, A. (1914). Bemerkung zu P. Harzers Abhandlung: Die Mitführung des Lichtes in Glas und die Aberration. Astron. Nachr., 199, 8-10.

Emelyanov, A. V., \& Emelyanov, I. A. (2015). Retrospective Analysis of the Well-Known Experiments. Int. J. Fund. Phys. Sci., 5, 1-11. https://doi.org/10.14331/ijfps.2015.330080

Faraday, M. (1846). Thoughts on Ray Vibrations. Phil. Mag., 28, N188.

Fiscaletti, D., \& Sorli, A. (2013). About a New Suggested Interpretation of Special Theory of Relativity within a Three-dimensional Euclid Space. Annales Universitatis Mariae Curie-Skłodowska Lublin-Polonia, LXVIII, 40-62. https://doi.org/10.2478/v10246-012-0018-1.

Fox, J. G. (1965). Evidence against Emission Theories. Am. J. Phys., 33, 1-17. https://doi.org/10.1119/1.1971219

Gaugett, A. (Ed.) (2014). The Sagnac Effect: 100 years later. Comptes Rendus Physique, 15, 787-916.

Gift, S. J. G. (2013). Light Transmission and the Sagnac Effect on the Rotating Earth. Applied Physics Research, 5, 93-106. https://doi.org/10.5539/apr.v5n5p93

Gift, S. J. G. (2014). Time Transfer and the Sagnac Correction in the GPS. Applied Physics Research, 6, 1-9. https://doi.org/10.5539/apr.v6n6p1

Gift, S. J. G. (2015). On the Selleri Transformations: Analysis of Recent Attempts by Kassner to Resolve Selleri's Paradox. Applied Physics Reseearch, 7, 112-120. https://doi.org/10.5539/apr.v7n2p112

Grøn, Ø. (1975). Relativistic Description of the Rotating Disk. Am. J. Phys., 43, 869-876.

Hadot, P. (2008). The Veil of Isis: An Essay on the History of the Idea of Nature. Belknap Press.

Hafele, J. C., \& Keating, R. E. (1972). Around-the World Atomic Clocks: Predicted Relativistic Time Gains. Science, 177, 168-170.

Hariharan, P. (1975). Sagnac or Michelson-Sagnac Interferometer? Applied Optics, 14, 2319-2321. 
Harress, F. (1912). The Speed of Light in Moving Bodies. PhD Thesis. Fridrich Schiller University, Jena, Germany.

Harzer, P. (1914). Über die Mitführung des Lichtes in Glas und die Aberration. Astron. Nachr., 198, 377-382. https://doi.org/10.1002/asna.19141982001

Harzer, P. (1920). Das Prinzip der schnellsten Ankunft des Lichtes und die Aberration. Astron. Nachr., 212, $65-$ 70. https://doi.org/10.1002/asna.19202120502

Heighway, J. (2017). Lorentz versus Einstein: A Proposal to Test the Existence of the Aether. International Journal of Theoretical and Mathematical Physics, 7, 21-24. https://doi.org/10.5923/j.ijtmp.20170702.01

Heras, R. (2015). Voigt's Transformations in the Beginning of the Relativistic Era. Retrieved from Arxiv.org $1411.2559 \mathrm{v} 3$

Ives, H. E. (1937). Graphical Exposition of the Michelson-Morley Experiment. J.O.S.A., 27, 177-180.

Ives, H. E. (1937). The Doppler Effect Considered in Relation to the Michelson-Morley Experiment. J.O.S.A., 27, 389-392.

Ives, H. E., \& Stilwell, G. R. (1938). An Experimental Study of the Rate of a Moving Atomic Clock. J.O.S.A., 28, 215-226.

Kassner, K. (2013). Ways to Resolve Selleri's Paradox. Retrieved from arxiv.org. 1302.6888v1

Knopf, O. (1920). Die Versuche von F. Harress über die Geschwindigkeit des Lichtes in bewegten Körpern. Ann. Phys. Vierte Folge. 62, 389-447.

Langevin, P. (1921). Sur la théorie de relativité et l'expérience de M. Sagnac. C.R. Acad. Sci., 173, 831-834.

Langevin, P. (1937). Sur l'expérience de M. Sagnac. C.R. Acad. Sci., 205, 304-306.

Laue, M. von (1911). Über einen Versuch zur Optik der bewegter Körper. Münchener Sitzungsberichte, 405-412.

Laue, M. von (1920). Zum Versuch von F. Harress. Annalen der Physik, 367, 448-463.

Lodge, O. (1893). Aberration Problems. Phil. Trans. Roy. Soc. of London, A, 727-804.

Malykin, G. B. (2000). The Sagnac Effect: Correct and Incorrect Explanations. Physics Uspekhi, 170, 1229-1252.

Malykin, G. B., \& Pozdnyakova, V. I. (2013). Ring Interferometry. (De Gruyter Studies in Mathamatical Physics Book 13), de Gruyter, Berlin.

Maraner, P., \& Zendri, J. P. (2012). General Relativistic Sagnac Formula Revised. Gen. Relativ. Gravit., 44, 17131723. https://doi.org/10.1007/s10714-012-1361-4

Marinov, S. (1974). The velocity of light is direction dependent. Czech. J. Phys. B, 24, 965-970. https://doi.org/10. 1007/BF01591047

Marquardt, P. (2013). A Propos SAGNAC. In Proceedings of the Natural Philosophy Alliance, $20^{\text {th }}$ Annual Conference, 10-13 July, 2013, U.S.A., 190-197.

Martínez, A. A. (2004). Ritz, Einstein, and the Emission Hypothesis. Phys. Perspect., 6, 4 - 28. https://doi.org/10.1007/s0016-003-0195-6

Maxwell, J. C. (1856). On Faraday's Lines of Force. Trans. Camb. Phil. Soc., 10, 155-188.

Michelson, A. A., \& Morley, E. W. (1887). On the Relative Motion of Earth and the Luminiferous Ether. Am. J. Sci., 34, 333-345.

Michelson, A.A., Gale, H. G., \& Person, F. (1925). The Effect of the Earth's Rotation on the Velocity of Light. Astrophys. J., 61, 137-145.

Nersessian, N. J. (1984). Faraday to Einstein: Constructing Meaning in Scientific Theories. Kluwe Academic Publishers, Dordrecht.

Norton, J. (2004). Einstein's Investigation of Galilean Covariant Electrodynamics Prior to 1905. Arch. Hist.Exact. Sci., 59, 45-105. https://doi.org/10.1007/s00407-004-0085-6

Pascoli, G. (2017). The Sagnac Effect and its Interpretation by Paul Langevin. C.R. Physique, 18, 563-569. https://doi.org/10.1016/j.crhy.2017.10.010

Pogany, B. (1928). Über die Wiederholung des Harressschen Versuches. Ann. Physik, 385, 217-231.

Post, E. J. (1967). Sagnac effect. Review of Modern Physics, 39, 475-493. 
Ritz, W. (1908). Recherches Critiques Sur L'Electrodynamique Générale. Annales de Chimie et de Physique, 13, 145-275.

Ritz, W., \& Einstein, A. (1909). Zum gegenwärtigen Stand des Strahlungsproblems. Phys. Zeit., 10, 323-324.

Rizzi, G., \& Ruggiero, M. L. (Eds.) (2004). Relativity in Rotating Frames. Fundamental Theories in Physics, 135. Springer.

Saba, M. M. F., \& Rosa, R. A. S. (2003). The Doppler Effect of a Sound Source Moving in a Circle. The Physics Teacher, 41, 89-91. https://doi.org/10.1119/1.1542044

Sagnac, G. (1913). L'éther lumineux démontré par l'effet du vent relative d'éther dans un interféromètre en rotation uniforme. C.R. Acad. Sci., 157, 708-710.

Sela, O., Tamir, B., Dolev, S., \& Elitzur, A. C. (2009). Can Special Relativity Be Derived from Galilean Mechanics Alone? Found. Phys., 39, 499-509. https://doi.org/10.1007/s10701-009-9301-1

Selleri, F. (1996). Noninvariant one-way velocity of light. Found. Phys., 26, 641-664.

Selleri, F. (2004). Sagnac effect: end of the mystery. In Relativity in rotating frames (pp. 57-77). Springer, Dordrecht.

Son, J. H., Kwon, M. H., \& Choi, H. M. (2014). Comparative Theoretical and Experimental Analyses of the Doppler Effect for a Sound Source Moving in a Circle. New Physics: Sae Mulli, 64, 162-169. https://doi.org/10.3938/NPSM.64.162

Spavieri, G. (2017). Testing Einstein's Second Postulate with an Experiment of the Sagnac Type. Phys. Astron. Int. J., 1,1 - 13. https://doi.org/10.15406/paij.2017.01.00003

Stewart, O. (1911). The Second Postulate of Relativity and the Electromagnetic Emission Theory of Light. Phys. Rev., 32, 418-428. https://doi.org/10.1103/physrevseriesi.32.418

Tartagli, A., \& Ruggiero, M. L. (2015). The Sagnac Effect and Pure Geometry. Am. J. Phys., 83, 427-432. https://doi.org/10.1119/1.4904319

Tolman, R. C. (1910). The Second Postulate of Relativity. Phys. Rev., 31, 26-40. https://doi.org/10.1103/ physreveriesi.31.26

Tolman, R. C. (1912). Some Emission Theories of Light. Phys. Rev., 35, 136-143. https://doi.org/10.1103/ physrevseriesi.35.136

Voigt, W. (1887). Über das Doppler'sche Princip. Nachr. Ges. Wiss. Göttingen, 8, 41-51.

Wang. R., Zheng, Y., Yao, A., \& Langley, D. (2003). Modified Sagnac Experiment for Measuring Travel-time Difference between Counterpropagating Light Beams in a Uniformly Moving Fiber. Physics Letters A, 312, 7-10.

Weinstein, G. (2015). Einstein's Pathway to the Special Theory of Relativity. Cambridge Scholars Publishing, Cambridge.

Witte, H. (1914). Der Sagnac- Effekt: ein Experimentum crusis zugunsten des Äthers? Verhandlungen der Deutschen Phys. Gesellschaft, 16, 142-150.

Witte, H. (1914). Sagnac Effect and Emission Theory. Verhandlungen der Deutschen Phys. Gesellschaft, 16, $755-$ 756.

\section{Copyrights}

Copyright for this article is retained by the author(s), with first publication rights granted to the journal.

This is an open-access article distributed under the terms and conditions of the Creative Commons Attribution license (http://creativecommons.org/licenses/by/4.0/). 